\title{
Sterile Pyuria in Patients Admitted to the Hospital With Infections Outside of the Urinary Tract
}

\author{
Jared B. Hooker, MS2, James W. Mold, MD, MPH, and Satish Kumar, MD
}

Objectives: The objective of this study was to determine the incidence, associations, evaluation, and management of pyuria in patients admitted to the hospital with nonurinary infections.

Methods: This study abstracted inpatient records of consecutive patients hospitalized for pneumonia, intra-abdominal infections, female genital tract infections (GYN infections), bacterial septicemia, and enteritis in the pediatric and adult medical and surgical units at an academic medical center.

Results: The study population included 210 patients (66 children; 144 adults). Nearly one-third had $\geq 5$ white blood cells (WBCs) per high-power field (pyuria). Pyuria was more common in women $(P<$ $.001)$ and in patients with GYN infections $(P=.001)$ and less common in patients with pneumonia $(P<$ $.001)$. Cultures were performed on 18 of 19 children (94.7\%) and 26 of 43 adults $(60.5 \%)$ with pyuria. of those, 11.1\% of children and 42.1\% of adults had a positive culture, and all but one of those met criteria for a urinary tract infection. Excluding patients with GYN infections, only $18.8 \%$ of patients with pyuria had a positive culture. Of the 44 patients with pyuria who were cultured, a positive culture was associated with having a GYN infection $(P=.01)$, moderate or large amounts of bacteria in the urine $(P=.005)$, and a positive urine nitrite $(P=.004)$. The absolute number of WBCs or red blood cells in the urine and the presence of casts, proteinuria, and leukocyte esterase were not associated with positive culture or urinary tract infection. Neither pyuria nor a positive culture was related to temperature, systemic WBC count, or serum albumin, blood urea nitrogen, or creatinine.

Conclusions: Sterile pyuria of uncertain cause is common in patients admitted to the hospital with acute nonurinary infections. (J Am Board Fam Med 2014;27:97-103.)

Keywords: Antibiotics, Fever, Patient Admission, Pyuria, Urinary Tract Infections

Urinalysis is a test commonly ordered at admission to a hospital, especially when the patient has a febrile illness. In most cases it provides useful information, but it can sometimes be misleading. One of the authors (JWM) has observed that patients admitted to the hospital with acute infectious illnesses unrelated to the urinary tract frequently

This article was externally peer reviewed.

Submitted 6 March 2013; revised 10 July 2013; accepted 15 July 2013.

From the College of Medicine (JBH), the Department of Family and Preventive Medicine (JWM), and the Division of Nephrology, Department of Internal Medicine (SK), University of Oklahoma Health Sciences Center, Oklahoma City.

Funding: Funding was provided by the Department of Family and Preventive Medicine, University of Oklahoma Health Sciences Center.

Conflict of interest: none declared.

Corresponding author: James W. Mold, MD, MPH, Department of Family and Preventive Medicine, University of Oklahoma Health Sciences Center, 900 NE 10th Street, Oklahoma City, OK 73104 (E-mail: james-mold@ouhsc.edu). have pyuria, which can confuse the admitting physicians, who sometimes alter their antibiotic choice based on the abnormal urinalysis even when the evidence for urinary tract infection (UTI) is weak.

Others have documented that sterile pyuria can occur in both adults and children with pneumonia and other acute febrile illness, suggesting that some feature of these illnesses or fever itself might cause leakage of white blood cells (WBCs) into the urine. $^{1-7}$ In fact, sterile pyuria has many causes (Table 1). ${ }^{1,8,9}$ However, the incidence, predictors, and clinical implications of pyuria in patients admitted to the hospital with infections outside of the urinary tract have not been studied. Believing that more information about this phenomenon might help physicians make better initial antibiotic choices, we undertook this study to estimate the incidence of pyuria at the time of hospital admission in patients with acute infections outside of the urinary tract and to gather information that could help clinicians 
Table 1. Some Reported Causes of Sterile Pyuria and the Tests Generally Used to Diagnose Them

\begin{tabular}{|c|c|}
\hline Cause & Evaluation \\
\hline \multicolumn{2}{|l|}{ Infectious causes } \\
\hline Perinephric abscess & Ultrasound; CT \\
\hline Renal tuberculosis & Urine TB culture and PCR \\
\hline Fungal infections of the kidneys & Gram stain; fungal culture \\
\hline Partially treated pyelonephritis & History; CT \\
\hline Fungal infections of the bladder & Gram stain; fungal culture \\
\hline Partially treated cystitis & History \\
\hline Contamination of urine with antiseptic & Repeat urinalysis \\
\hline Prostatitis & Prostate exam \\
\hline Urethritis & History; urine PCR; chlamydia culture \\
\hline Cervicitis & Vaginal exam; cervical culture \\
\hline Vaginitis & Vaginal exam; wet prep/KOH \\
\hline Appendicitis, diverticulitis & US; CT \\
\hline Q fever & History; serology \\
\hline \multicolumn{2}{|l|}{ Noninfectious causes } \\
\hline Crystal nephropathy and nephrolithiasis & CT, IVP \\
\hline Lithium or heavy metal nephropathy & History; lithium level; heavy metal tests \\
\hline \multicolumn{2}{|c|}{ Renal papillary necrosis (diabetes, sickle cell disease, analgesic nephropathy) } \\
\hline Renal sarcoidosis & Urinalysis, IVP; US; CT \\
\hline Polycystic kidney disease & Renal biopsy \\
\hline Renal transplant rejection & US; CT \\
\hline Interstitial nephritis & History; renal biopsy \\
\hline Genitourinary malignancy & Urine eosinophils, renal biopsy \\
\hline Interstitial cystitis & CT; cystoscopy \\
\hline Systemic lupus erythematosis, other autoimmune diseases & Cystoscopy \\
\hline \multirow[t]{2}{*}{ Kawasaki disease } & ESR; ANA, anti-DNA \\
\hline & History; physical exam \\
\hline
\end{tabular}

ANA, antinuclear antibodies; CT, computed tomography; ESR, erythrocyte sedimentation rate; KOH, potassium hydroxide; IVP, intravenous pyelogram; PCR, polymerase chain reaction; TB, tuberculosis; US, ultrasound.

make better initial evaluation and treatment decisions in such cases.

We specifically set about to answer the following questions about patients admitted to the hospital with non-urinary tract infections: (1) What are the incidences of pyuria, bacteriuria, and UTI? (2) What clinical factors are associated with pyuria in these patients? and (3) How often and in what ways does pyuria impact management decisions?

\section{Methods}

\section{Data Collection}

From a list of consecutive patients (both adults and children) discharged from the medical and surgical services of an academic medical center between June 1 and September 30, 2011, we retrospectively identified and abstracted the medical records of all patients with the following diagnoses at discharge: (1) pneumonia, (2) bacterial septicemia, (3) intra- abdominal infection, (4) enteritis, or (5) female genital tract (GYN) infections. We chose to look at patients with pneumonia and septicemia because prior studies have suggested a link between these infections and sterile pyuria, sometimes referred to as "febrile pyuria." 1,2 Intra-abdominal infections, female GYN infections, and enteritis were selected because they might cause external inflammation of the urinary tract. We excluded patients with diagnoses of end-stage renal disease, genitourinary stones, sickle cell disease, malignant hypertension, and sarcoidosis-conditions known to cause sterile pyuria - at admission or discharge. We also excluded patients who did not have a urinalysis within 24 hours of admission, lacked a history and physical examination in their record, or had an indwelling catheter at the time of admission.

After orientation to the electronic medical record (MediTech, Westwood, MA) and abstraction 
training, one author (JBH) abstracted the data from the medical records into a spreadsheet (Microsoft Excel; Microsoft Corp., Redmond, WA) and then imported them into a statistical analysis program (Statistix 9; Analytical Software, Tallahassee, FL). A subset of $10 \%$ of the records was reviewed by another author (JWM) to check the accuracy of the abstractions and direct further training. The abstractions captured demographic data (age, race, sex, primary insurance type, habitat before admission); weight and temperature at admission; presenting symptoms (categorized by organ system); specific urinary symptoms and signs (frequency, dysuria, urgency, incontinence, flank pain, abdominal pain, lower abdominal tenderness, and costovertebral angle [CVA] tenderness); WBC count; serum creatinine, blood urea nitrogen (BUN), and albumin levels; urinalysis and urine culture results at admission; method of urine collection; results of last urinalysis before discharge; and antibiotics given to the patient during the first 24 hours after admission. In addition, we noted whether any other studies were done that could have been used to determine the cause of sterile pyuria (urine test for eosinophils, urine culture for tuberculosis, ultrasound of the kidneys/bladder, intravenous pyelogram, renal radionucleotide scan, cystoscopy, magnetic resonance imaging of the kidneys, computed tomography of the abdomen). Glomerular filtration rate (GFR) was estimated using both the Cockroff-Gault (CG) and the Modification of Diet in Renal Disease (MDRD) equations. ${ }^{10}$

For urine protein, nitrite, ketones, squamous cells, and casts, we recorded only whether the result was negative or positive. To quantify the number of WBCs and red blood cells per high-powered field (HPF), we used the categories reported by the hospital's laboratory $(0-2,2-5,5-10,10-15,15-$ $20,20-25,25-30,30-40,40-50,50-100,>100$, or too numerous to count). For numbers of bacteria in the urine, we used the categories of none, light, moderate, or heavy, as reported by the laboratory. For the urine culture results, we used the categories reported by the hospital's microbiology laboratory ( $<100$ or no growth; 100-9999; 10,000-49,999; 50,000-99,999; and $\geq 100,000$ colony-forming units $/ \mathrm{mL}$, as well as multiple organisms). Antibiotics given during the first 24 hours of hospitalization were recorded and categorized by class.

We defined "pyuria" as $>5 \mathrm{WBC} / \mathrm{HPF}$. A positive urine culture was defined as at least 100,000 colony-forming units/mL of a single bacterial species if the urine was obtained using the clean catch (CC) method and at least 50,000 colony-forming units $/ \mathrm{cm}^{3}$ of a single bacterial species if the urine was obtained by catheterization (cath). Urine cultures with multiple bacterial species were considered negative. We defined sterile pyuria as $>5$ $\mathrm{WBCs} / \mathrm{HPF}$ with a negative urine culture. A UTI was defined as the presence of a positive urine culture and one or more of the following urinary symptoms or signs: frequency, dysuria, urgency, incontinence, flank pain, abdominal pain, lower abdominal tenderness, or CVA tenderness.

The study was approved by the University of Oklahoma Health Sciences Center Institutional Review Board, and a Health Insurance Portability and Accountability Act waiver was granted for our abstraction of private health information to be deidentified after review.

\section{Statistical Analyses}

We first calculated descriptive statistics for all variables across all patients (Table 2) and then compared the proportions of patients with pyuria, pyuria with a positive urine culture, and urinary tract infection in children versus adults, males versus females, and across diagnostic groups (Table 3). Because of the relatively small numbers of patients in each subcategory, we used Fisher's exact test for the bivariate analyses, and we chose not to construct regression models. Because of multiple comparisons, we set our $\alpha$ at $1 \% ; P<.01$ was considered significant.

Next we looked at patients with communityacquired pneumonia and sterile pyuria to see whether initial antibiotic choice was altered as a result of the urine findings. We also examined all cases of sterile pyuria to see whether additional investigations were conducted to determine the potential cause of the pyuria. Finally, we examined associations between sterile pyuria and initial body temperature, circulating WBC count, serum albumin, BUN, creatinine, and estimated GFR in an attempt to identify possible causes of the pyuria in the absence of UTI.

\section{Results}

During the study period, 1216 patients were discharged from the acute medical and surgical services of the Oklahoma Medical Center with a pri- 
Table 2. Characteristics of the Study Population

(n= 210)

\begin{tabular}{|c|c|}
\hline Population Characteristics & No. (\%) \\
\hline \multicolumn{2}{|l|}{ Age (years) } \\
\hline$<18$ & $66(31.4)$ \\
\hline$\geq 18$ & $144(68.6)$ \\
\hline \multicolumn{2}{|l|}{ Sex } \\
\hline Female & $116(55.2)$ \\
\hline Male & $94(44.8)$ \\
\hline \multicolumn{2}{|l|}{ Race } \\
\hline White & $165(78.6)$ \\
\hline Black & $29(13.8)$ \\
\hline American Indian & $10(4.8)$ \\
\hline Other/unknown & $6(2.9)$ \\
\hline \multicolumn{2}{|l|}{ Primary health insurance } \\
\hline Medicare & $54(25.8)$ \\
\hline Medicaid & $63(30.1)$ \\
\hline Commercial & $50(23.9)$ \\
\hline Uninsured & $42(20.1)$ \\
\hline \multicolumn{2}{|l|}{ Setting before admission } \\
\hline Home & $171(81.4)$ \\
\hline Nursing home & $9(4.3)$ \\
\hline Inpatient facility & $26(12.4)$ \\
\hline Unknown & $4(1.9)$ \\
\hline \multicolumn{2}{|l|}{ Primary discharge diagnosis } \\
\hline Pneumonia & $45(19.5)$ \\
\hline Intra-abdominal infection & $88(41.9)$ \\
\hline Female genital infection & $10(4.8)$ \\
\hline Enteritis & $41(19.5)$ \\
\hline Septicemia, bacterial & $26(12.4)$ \\
\hline \multicolumn{2}{|l|}{ Pyuria } \\
\hline \multicolumn{2}{|l|}{$>5 \mathrm{WBCs} / \mathrm{HPF}$} \\
\hline No & $148(70.5)$ \\
\hline Yes & $62(29.5)$ \\
\hline \multicolumn{2}{|l|}{$>10 \mathrm{WBCs} / \mathrm{HPF}$} \\
\hline No & $179(85.2)$ \\
\hline Yes & $31(14.8)$ \\
\hline \multicolumn{2}{|c|}{ Urine culture result (if urine cultured) } \\
\hline Negative & $31(70.5)$ \\
\hline Positive & $13(29.5)$ \\
\hline \multicolumn{2}{|c|}{ At least 1 possible urinary tract symptom } \\
\hline No & $30(14.3)$ \\
\hline Yes & $180(85.7)$ \\
\hline \multicolumn{2}{|l|}{ Urinary tract infection } \\
\hline No & $198(94.3)$ \\
\hline Yes & $12(5.7)$ \\
\hline
\end{tabular}

HPF, high-powered field; WBC, white blood cells.

mary diagnosis of an acute infectious disease. Of those, 386 had 1 of the 5 qualifying infectious diseases. We excluded 155 patients for whom urinalysis was not performed within 24 hours of ad- mission, 10 patients who had an indwelling urinary catheter at the time of admission, and 1 patient who had a ureterostomy tube. In 4 other cases we could not find a history and physical examination at admission, and in 6 cases the diagnosis of pneumonia at discharge was not supported by clinical and laboratory findings. The remaining 210 patients constituted the study sample. Among study patients, two-thirds $(68.6 \%)$ were adults, and slightly more than half were women $(55.2 \%)$. Other patient characteristics can be found in Table 2 .

\section{Incidence and Predictors of Pyuria, Sterile Pyuria, and UTI at Admission}

Nearly one third of both adults (43 of 144; 29.9\%) and children (19 of $66 ; 29 \%$ ) had pyuria at admission to the hospital. In 18 of the 19 children (95\%) and 26 of the 43 adults (60\%) with pyuria, urine cultures were performed. Among the 44 patients with pyuria who had been cultured, 13 (29.5\%) had a positive culture, 12 of whom had at least one urinary tract symptom or sign, so 2 of 18 children $(11.1 \%)$ and 10 of 26 adults $(38.5 \%)$ with pyuria met criteria for UTI (see Tables 2 and 3).

Children with pyuria were more likely to be cultured but less likely to have a positive culture than adults. Females were more likely to have pyuria than men $(43.1 \%$ vs $12.8 \% ; P<.0001)$, but they were neither more nor less likely to have a positive culture or a UTI. There were small, statistically nonsignificant differences in the rates of pyuria and positive cultures by urine collection method (pyuria: $29.8 \%$ for the CC method vs $20 \%$ for cath; positive culture: $22.6 \%$ for the CC method vs $33.3 \%$ for cath, respectively). Female patients with GYN infections were more likely than patients with the other 4 types of infections to have pyuria, a positive urine culture, and a UTI. The rate of UTI in those patients was $50 \%$ (see Table 3). For all other patients with pyuria, the probabilities of a positive culture and UTI were both $18.8 \%$.

In the 44 patients who had pyuria who were cultured, a positive culture was associated with having a GYN infection $\left(\chi^{2} 6.57 ; P=.01\right)$, having moderate or large amounts of bacteria in the urine $\left(\chi^{2} 7.74 ; P=.005\right)$, and having a urine culture positive for nitrite $\left(\chi^{2} 8.19 ; P=.004\right)$. The absolute number of WBCs or red blood cells in the urine, the presence of casts of any kind, proteinuria, and 
Table 3. Documented Pyuria and Positive Cultures by Age, Sex, Weight, and Diagnosis

\begin{tabular}{|c|c|c|c|c|}
\hline Characteristics & Pyuria & $P$ & Culture Positive* & $P$ \\
\hline All Patients & $62 / 210(29.5)$ & & $13 / 44(29.5)$ & \\
\hline Age group & & .87 & & .09 \\
\hline Children & $19 / 66(28.8)$ & & $2 / 18(11.1)$ & \\
\hline Adults & $43 / 144(29.9)$ & & $11 / 26(42.3)$ & \\
\hline Sex & & $<.001$ & & .69 \\
\hline Female & $50 / 116(43.1)$ & & $10 / 36(27.8)$ & \\
\hline Male & $12 / 94(12.8)$ & & $3 / 8(37.5)$ & \\
\hline Weight (lb) & & .61 & & .26 \\
\hline$<100$ & $13 / 45(28.9)$ & & $1 / 13(7.7)$ & \\
\hline $100-200$ & $37 / 111(33.3)$ & & $8 / 23(34.8)$ & \\
\hline$>200$ & $9 / 36(25)$ & & $3 / 6(50)$ & \\
\hline \multicolumn{5}{|l|}{ Primary diagnosis } \\
\hline Pneumonia & $4 / 45(8.9)$ & $<.001$ & $0 / 3(0)$ & .54 \\
\hline Septicemia & 28/88 (31.8) & .54 & 3/17 (17.6) & .20 \\
\hline Intra-abdominal infect. & $12 / 41(29.3)$ & 1.00 & $1 / 10(10)$ & .24 \\
\hline Enteritis & $3 / 10(33)$ & 1.00 & $2 / 2(100)$ & .08 \\
\hline Female genital infection & $15 / 26(57.7)$ & .001 & $7 / 12(58.3)$ & .02 \\
\hline \multicolumn{5}{|l|}{ Signs and symptoms } \\
\hline Fever & 26/93 (28) & .76 & $5 / 23(21.7)$ & .33 \\
\hline Abdominal pain & $43 / 126(34.1)$ & .09 & $9 / 30(30)$ & 1.00 \\
\hline Flank pain & $1 / 6(16.7)$ & .67 & $0 / 1(0)$ & 1.00 \\
\hline Abdominal tenderness & $29 / 86(33.7)$ & .28 & $3 / 21(14.3)$ & .05 \\
\hline CVA tenderness & $1 / 3(33)$ & 1.00 & $0 / 1(0)$ & 1.00 \\
\hline \multicolumn{5}{|l|}{ Laboratory tests } \\
\hline Urine nitrite & $14 / 19(73.7)$ & $<.001$ & 7/11 (63.6) & .008 \\
\hline Urine bacteria $(\geq \bmod$.) & $30 / 44(68.2)$ & $<.001$ & $11 / 23(47.8)$ & .008 \\
\hline eGFR by $\mathrm{CG}<60$ & $16 / 48(33.3)$ & .56 & $3 / 13(23.1)$ & .23 \\
\hline
\end{tabular}

${ }^{*}$ Values are $\mathrm{n} /$ numbers of patients with pyuria who had a urine culture performed (\%).

All $P$ values were obtained using Fisher's exact test.

CG, Cockroff-Gault; CVA, costovertebral angle; eGFR, estimated glomerular filtration rate.

positive leukocyte esterase were not associated with a positive culture or UTI.

\section{Effect of Sterile Pyuria on Management Decisions}

There were 27 cases of community-acquired pneumonia. However, only 2 had pyuria. In one of those cases, the choice of antibiotic was altered because of the pyuria. It was impossible to tell whether decision making was affected in the other diagnostic groups because of the frequent need to cover for Gram-negative pathogens for other reasons. Repeat urinalyses were performed on 35 of the 62 patients with pyuria $(56.5 \%)$ and 6 of the $31 \mathrm{pa}-$ tients $(19.4 \%)$ with sterile pyuria. In those 6 cases, 3 of the follow-up urinalyses $(50 \%)$ showed no pyuria.

In 133 of the 210 cases $(63.3 \%)$, at least one other test had been ordered that could have, at least in part, been in response to the abnormal urinalysis at admission. These included abdominal computed tomography, ultrasound of the kidneys or bladder, magnetic resonance imaging of the kidneys, intravenous pyelogram, renal radionucleotide scan, urine for eosinophils, and urine culture for tuberculosis.

\section{Potential Causes of Sterile Pyuria}

Sterile pyuria was unrelated to reported fever, measured body temperature, systemic WBC count, or serum albumin. It was, however, associated with lower estimated GFR (mean of $63.1 \mathrm{~mL} / \mathrm{min}$ in patients with sterile pyuria vs mean of $96.3 \mathrm{~mL} / \mathrm{min}$ in others; odds ratio, 0.99 ; $95 \%$ confidence interval, $0.97-1.00)$. This effect was lost when estimated GFR was dichotomized (see Table 3). The mean BUN and creatinine levels were also somewhat 
higher in patients with sterile pyuria (means of 20.2 vs 17.1 and 1.25 vs 1.05 , respectively), but these differences were not statistically significant.

\section{Discussion}

Pyuria was common among patients admitted to an acute care hospital who were ultimately found to have infections outside of the urinary tract as the reason for hospitalization. Aside from female patients with GYN infections, pyuria was unlikely to be caused by a UTI, and it should be noted that the proportion of patients with pyuria who had a UTI is likely to be overestimated. The prevalence of bacterial colonization of the urinary tract is $10 \%$ to $50 \%$ in adults, depending on age, sex, and frailty, and $5 \%$ among febrile children with positive urine cultures. ${ }^{11,12}$ Some the qualifying symptom(s) used in our study (eg, abdominal pain or tenderness) could have been caused by the primary diagnoses.

Choice of antibiotic at admission remains a matter of clinical judgment. Our findings inform that judgment by demonstrating that the presence of pyuria does not usually indicate the presence of a UTI in patients with pneumonia, bacterial septicemia, intra-abdominal infection, and enteritis. These findings suggest that when it is clear that the primary infection is not in the urinary tract, clinicians should not feel obligated to alter their choice of antibiotics unless there is good reason to believe the patient has more than one infection. Gynecological infections seem to be a special case. These infections could cause inflammation of the external surfaces of the urinary organs or contiguous infection, potentially resulting in pyuria and sometimes a UTI.

Our study also produced other interesting findings, which tended to challenge established dogma. First, because of difficulties involved in obtaining CC urine specimens and the potential for the contamination of urine specimens with WBCs of vaginal origin, women were expected to have a higher rate of both pyuria and sterile pyuria. In our study, women did have a higher incidence of pyuria but the proportion with positive cultures was not different between women and men with pyuria.

Second, we also expected that CC urine tests would be more likely than urine samples obtained by catheter to have $>5 \mathrm{WBCs} / \mathrm{HPF}$ and to grow multiple organisms. While there were small differences, they were not statistically significant. Third, we were surprised at the absence of a strong correlation between pneumonia and pyuria, since this association was reported in the literature and was one of the clinical observations leading to this study. In fact, in this sample, pneumonia was the least likely of the 5 infections to be associated with pyuria.

The high incidence of sterile pyuria in these patients raises questions about the need for further work-up. Approximately 50\% no longer had pyuria on a repeat urinalysis, so repeating the urinalysis would seem to be a logical first step. If the pyuria persists, a list of probable causes and diagnostic tests should be considered (Table 1).

We speculated that there might be associations between pyuria and both fever and systemic WBC count. However, we found neither. In patients with pyuria, those with kidney damage (lower estimated GFR) were more likely to have a negative culture. The latter association was seen only for the CG estimated GFR and not for the MDRD estimate. Sterile pyuria has been reported in patients with end-stage renal disease ${ }^{13,14}$ but to our knowledge it has not been reported in patients with earlier stages of chronic kidney disease.

The study has several important limitations. Most significant is the relatively small sample size, which reduced our power to detect some risk factors and predictors in particular within the sex and age subgroups. Data were abstracted by a medical student before his clinical rotations. While he received training and another author (JWM) re-abstracted a subset of records, it is possible that some classification errors may have occurred.

Another potential weakness was selection of patients based on diagnosis at discharge. It is likely that in some cases the final diagnoses were not clear at admission and that UTI was in the differential at that point. However, we do not believe that this substantially changes our conclusions or recommendations. We also believe that excluding patients with conditions known to cause sterile pyuria-thus reducing the numerator of the proportion — strengthens our conclusions.

The exclusion of patients who did not have a urinalysis at admission may have introduced bias. If so, it would have been in a direction supportive of our conclusions because UTI would have been less likely in patients in whom UTI was not suspected by the admitting physician. A routine urinalysis in 
all patients would have reduced the percentage of positive urine cultures.

Because the involved hospital is a teaching hospital, the patients may have been younger and more complex than those typically admitted to other acute care hospitals, potentially reducing generalizability.

A substantial number of patients with pyuria did not have urine cultures, in part because of the failure of the emergency department physicians to order reflexive urine cultures, a fact not always appreciated by the admitting teams. It is impossible to say how this might have affected our results. It is possible that some patients had received antibiotics either at home or before producing their urine samples for testing. This would have reduced the rate of bias of positive urine cultures on our results and conclusions. Genitourinary infections with chlamydia and other atypical bacteria would be expected to produce pyuria and negative cultures since these organisms do not grow on standard culture media.

Some may question our choice of $>5$ WBCs/ HPF. When we recalculated the statistics using $>10 \mathrm{WBCs} / \mathrm{HPF}$, the results were nearly identical. Others may be concerned that we set culture positivity at $>50,000$ colonies/cc in patients whose urine was obtained by catheter since some have suggested the cutoff should be at $>10,000$ colonies/ cc. In this study it would have made no difference because only one patient with pyuria had a catheterized specimen with a bacterial colony count of between $>10,000$ and $<50,000$.

Despite these limitations, we believe that our study adds new information in this area of limited literature and helps clinical decision making in the choice of antibiotics for patients with infections outside the urinary tract. A great deal more work certainly needs to be done in this area.

\section{References}

1. Dieter RS. Sterile pyuria: a differential diagnosis. Compr Ther 2000;26:150-2.

2. Goldring W. Studies of the kidney in acute infection: III. Observations with the urine sediment count (Addis) and the urea clearance test in Lobar pneumonia. J Clin Invest 1931;10:355-67.

3. Turner GM, Coulthard MG. Fever can cause pyuria in children. BMJ 1995;311:924.

4. North AF. Bacteriuria in children with acute febrile illnesses. J Pediatr 1963;63:408-11.

5. Shike H, Kanegaye JT, Best BM, Pancheri J, Buns JC. Pyuria associated with acute Kawasaki disease and fever from other causes. Pediatr Infect Dis J 2009;28:440-3.

6. Hogg RJ. A search for the "clusive" urinary tract infection in febrile infants. Pediatr Infect Dis J 1987; 6:233-4.

7. Wu CY, Chiou YH, Wang RS, Huang WC, Lee WY, Chiou CC. Prolonged fever and pyuria: a urinary tract infection presentation of incomplete $\mathrm{Ka}-$ wasaki disease. Acta Paediatr 2005;94:375-7.

8. Dogunro AS. A comparative study of pyuria and asymptomatic bacteruria in school children. Trop Doct 1991;21:26-8.

9. Gleckman R, Esposito A. Sterile pyuria in the elderly. Am Fam Physician 1979;19:109-11.

10. Levey AS, Stevens LA, Schmid CH, et al. A new equation to estimate glomerular filtration rate. Ann Intern Med 2009;150:604-12.

11. Colgan R, Nicolle LE, McGlone A, Hooton TM. Asymptomatic bacteriuria in adults. Am Fam Physician 2006;74:985-90.

12. Hoberman A, Wald ER, Reynolds EA, Penchansky L, Charron M. Pyuria and bacteriuria in urine specimens obtained by catheter from young children with fever. J Pediatr 1994;124:513-9.

13. Eisinger RP, Asghar F, Kolasa C, Weinstein MP. Does pyuria indicate infection in asymptomatic dialysis patients? Clin Nephrol 1997;47:50-1.

14. Cabaluna CC, Gary NE, Eisinger RP. Urinalysis in patients on chronic hemodialysis. Urology 1977;10: $103-4$. 